\title{
Some Special Conditions in Topological Summed
}

\author{
Arife Atay $^{1}$, H. İlhan Tutalar ${ }^{2}$ \\ Department of Mathematics, Faculty of Science, Dicle University, Diyarbakır, Turkey ${ }^{1,2}$
}

\begin{abstract}
This study includes free union of a disjoint non-empty collection of topological spaces and research on the disjoint union topology (topological summed). Definitions, theorems and some results for topological summed have been obtained by using the known definitions and theorems for the topological spaces.
\end{abstract}

Keywords: Free Union, Disjoint Union Topology, Topological Summed, Topology.

\section{INTRODUCTION}

Let $\left\{\left(\mathrm{X}_{\lambda}, \tau_{\lambda}\right)\right\}_{\lambda \in \Lambda}$ be a collection of topological space. Let For every subfamily $\left\{\mathrm{T}_{1}, \mathrm{~T}_{2}, \ldots\right\} \subseteq \tau$ and for each $\lambda \in \Lambda$ we $(\mathrm{X}, \tau)$ be a topological space with $\mathrm{X}=U \mathrm{X}_{\lambda}, \lambda \in \Lambda$. $(\mathrm{X}, \tau)$ have, is a free union of $\left(X_{\lambda}, \tau_{\lambda}\right)$ iff the following condition $\left(U_{i} T_{i}\right) \cap X_{\lambda}=U_{i}\left(T_{i} \cap X_{\lambda}\right) \in \tau_{\lambda}$ satisfied $\mathrm{T} \in \tau \Leftrightarrow \forall \lambda \in \Lambda, \mathrm{T} \cap \mathrm{X}_{\lambda} \in \tau_{\lambda}$ and hence $\left(U_{i} T_{i}\right) \in \tau$

If $\left(X_{\lambda}, \tau_{\lambda}\right)$ is subspace of $(X, \tau)$ and $X_{\lambda}$ is open in $X$ for Theorem 2.2: For each $\lambda \in \Lambda$, $\tau_{\lambda}$ is subfamily of $\tau$. every $\lambda \in \Lambda,(X, \tau)$ is a free union of the subspaces Proof: Let $A \in \tau_{\lambda}$. Then we have $A \cap X_{\lambda}=A \in \tau_{\lambda}$. Also $\left(\mathrm{X}_{\lambda}, \tau_{\lambda}\right)$. But the spaces $\left(\mathrm{X}_{\lambda}, \tau_{\lambda}\right)$ are not necessary to be a subspace of $X$. So we will give the definition of disjoint union topology and topological summed and investigate some of results about topological summed in this paper.

Let $\left\{\left(\mathrm{X}_{\lambda}, \tau_{\lambda}\right)\right\}$ be a disjoint non-empty collection of topological spaces $\left(X_{\lambda}, \tau_{\lambda}\right)$ indexed by a set $\Lambda$. The disjoint union $\mathrm{X}=U \mathrm{X}_{\lambda}, \lambda \in \Lambda$ is a topological space with the following topology

$\tau=\left\{\mathrm{T} \subseteq \mathrm{X}: \mathrm{T} \cap \mathrm{X}_{\lambda} \in \tau_{\lambda}\right.$ for each $\left.\lambda \in \Lambda\right\}$

$\tau$ is a disjoint union topology and $X$ is a topological summed of a disjoint non-empty collection of topological spaces $X_{\lambda}$. Hence, $(X, \tau)$ is a free union of $\left(X_{\lambda}, \tau_{\lambda}\right)$.

for $\lambda \neq \lambda^{\prime}, A \cap X_{\lambda^{\prime}}=\emptyset \in \tau_{\lambda}$. Hence, we obtain $A \cap X_{\lambda} \in$ $\tau_{\lambda}$, for each $\lambda \in \Lambda$ and so $A \in \tau$.

Example 2.1:Let $\left\{\left(\mathrm{X}_{\lambda}, \tau_{\lambda}\right)\right\}_{\lambda \in \Lambda}$ be a disjoint non-empty collection of topological spaces. It is clear that $U_{i \in I} T_{i} \in \tau$ for each $\lambda \in \Lambda, \mathrm{T}_{\mathrm{i}} \in \tau_{\lambda}$. Also by theorem 2.2 we obtain $\tau=\left\{\bigcup_{i \in I} T_{i}: \exists \lambda \in \Lambda, T_{i} \in \tau_{\lambda}\right\}$.

Suppose now that $\tau_{\lambda}$ is indiscrete topology. For every $\lambda \in \Lambda$, we have $X_{\lambda} \in \tau$. Hence, $\tau$ can not be indiscrete topology except $\Lambda=\{1\}$.

Theorem 2.3: $\tau$ is indiscrete iff $\Lambda=\{1\}$.

Proof: It is obtained from the above example easily.

In this paper we understand $\left\{\left(X_{\lambda}, \tau_{\lambda}\right)\right\}_{\lambda \in \Lambda}$ is the disjoint non-empty collection of topological spaces indexed by a set $\Lambda$ and $(X, \tau)$ is the topological summed of collection $\left\{\left(\mathrm{X}_{\lambda}, \tau_{\lambda}\right)\right\}_{\lambda \in \Lambda}$.

Now we will give free union of a disjoint non-empty collection of topological spaces. Definitions, theorems and some results for topological summed have been obtained by using the known definitions and theorems for the topological spaces [1], [2], [3].

\section{MAIN RESULTS}

Our definition to be used in the future is the following.

Definition 2.1: For $\left(x_{n}\right)_{\mathbb{N}}$ sequence of $X$ and $b \in X$, $(\mathrm{x})_{\mathrm{n}} \rightarrow \mathrm{b} \Leftrightarrow \exists ! \lambda \in \Lambda, \exists \mathrm{N}_{\mathrm{k}} \in \mathbb{N}: \mathrm{n} \geq \mathrm{N}_{\mathrm{k}} \Rightarrow \mathrm{x}_{\mathrm{n}} \in \mathrm{T}^{(\mathrm{k})}$ every $\mathrm{T}^{(\mathrm{k})} \in \tau_{\lambda}$ containing $\mathrm{b}$.

Theorem 2.1: If $\tau$ is the family in the sense of definition in Introduction, then $(\mathrm{X}, \tau)$ is a topological space.

Proof: For each $\lambda \in \Lambda, \emptyset \cap X_{\lambda}=\emptyset \in \tau_{\lambda} \Rightarrow \emptyset \in \tau_{\lambda}$, $\mathrm{X} \cap \mathrm{X}_{\lambda}=\mathrm{X}_{\lambda} \in \tau_{\lambda} \Rightarrow \mathrm{X} \in \tau_{\lambda}$.

For every finite subfamily $\left\{\mathrm{T}_{1}, \mathrm{~T}_{2}, \ldots, \mathrm{T}_{\mathrm{r}}\right\} \subseteq \tau$, we have

Theorem 2.4: $\tau$ is discrete iff for each $\lambda \in \Lambda, \tau_{\lambda}$ is discrete.

Proof: If $\tau$ is discrete, $\{\mathrm{x}\}$ is element of $\boldsymbol{\tau}$, for each $\mathrm{x} \in \mathrm{X}$. Then $\{x\} \cap X_{\lambda}$ is element of $\tau_{\lambda}$, for each $\lambda \in \Lambda$. As $X_{\lambda}$ is discrete, $x \in X$ is element of $X_{\lambda}$ for only one $\lambda \in \Lambda$, i.e., for $\lambda \neq \lambda^{\prime}, \mathrm{x} \in \mathrm{X}_{\lambda} \Rightarrow\{\mathrm{x}\} \cap \mathrm{X}_{\lambda^{\prime}}=\emptyset$ and $\{\mathrm{x}\} \cap \mathrm{X}_{\lambda}=\{\mathrm{x}\} \in$ $\tau_{\lambda}$. So $\tau_{\lambda}$ contain all single point set, for each $\lambda \in \Lambda$. So $\tau_{\lambda}$ is discrete topology.

Conversely, if $\tau_{\lambda}$ is discrete, $\tau$ is discrete because of $\tau_{\lambda} \subseteq \tau$

Theorem 2.5: $\mathrm{X}_{\lambda}$ is subspace of $\mathrm{X}$.

Proof: Because of $\mathrm{X}=\bigcup_{\lambda \in \Lambda} \mathrm{X}_{\lambda}, \mathrm{X}_{\lambda}$ is subset of $\mathrm{X}$. However, for every $\lambda \in \Lambda$ and for $\tau^{\prime}=\left\{\mathrm{X}_{\lambda} \cap \mathrm{T}\right.$ : T $\left.\in \tau\right\}$ we must show that $\tau_{\lambda}=\tau^{\prime}$.

$$
\begin{array}{r}
\mathrm{T} \in \tau^{\prime} \Rightarrow \exists \mathrm{V} \in \tau: \mathrm{T}=\mathrm{X}_{\lambda} \cap \mathrm{V} \stackrel{\mathrm{V} \in \tau}{\Rightarrow} \mathrm{X}_{\lambda} \cap \mathrm{V} \in \tau_{\lambda} \Rightarrow \mathrm{T} \in \tau_{\lambda} \\
\mathrm{U} \in \tau_{\lambda} \stackrel{\tau_{\lambda} \subseteq \tau}{\Longrightarrow} \mathrm{U} \in \tau \stackrel{\mathrm{U} \subseteq \mathrm{X}_{\lambda}}{\Longrightarrow} \mathrm{U}=\mathrm{U} \cap \mathrm{X}_{\lambda}, \mathrm{U} \in \tau \Rightarrow \mathrm{U} \in \tau^{\prime}
\end{array}
$$

Then we obtain $\tau_{\lambda}=\tau^{\prime}$

Theorem 2.6: If $A \cap X_{i}=\{x\}$ and $\{x\} \notin \tau_{i}$, for $x \in X$ and $i \in \Lambda$. A can't be neighborhood of $x$.

$\left(\bigcap_{i=1}^{r} T_{i}\right) \cap X_{\lambda}=\bigcap_{i=1}^{r}\left(T_{i} \cap X_{\lambda}\right)$ for each $\lambda \in \Lambda$. Since Proof: Since $\left\{\left(X_{\lambda}, \tau_{\lambda}\right)\right\}_{\lambda \in \Lambda}$ is the disjoint collection of $\mathrm{T}_{\mathrm{i}} \in \tau$, for every $\lambda \in \Lambda$ we have $\mathrm{T}_{\mathrm{i}} \cap \mathrm{X}_{\lambda} \in \tau_{\lambda}$ and we know topological spaces, $\mathrm{X}_{\mathrm{i}}$ is the only subset such that $\mathrm{x} \in \mathrm{X}_{\mathrm{i}}$. $\left(X_{\lambda}, \tau_{\lambda}\right)$ is topological space so $\bigcap_{i=1}^{r}\left(T_{i} \cap X_{\lambda}\right) \in \tau_{\lambda}$. Then On the contrary, $A$ is neighborhood of $x$ such that $A \cap$ $\bigcap_{i=1}^{r}\left(T_{i} \cap X_{\lambda}\right)=\left(\cap_{i=1}^{r} T_{i}\right) \cap X_{\lambda} \in \tau_{\lambda}$ and hence $\bigcap_{i=1}^{r} T_{i} \in X_{i}=\{x\}$ and $\{x\} \notin \tau_{i}$. In this case, there is $U \in \tau$ such that $\tau$. $\mathrm{x} \in \mathrm{U} \subseteq \mathrm{A}$. So $\mathrm{U} \cap \mathrm{X}_{\lambda} \in \tau_{\lambda}$, for each $\lambda \in \Lambda$, i.e., $\mathrm{U} \cap \mathrm{X}_{\mathrm{i}}=$ 
$\{\mathrm{x}\} \in \tau_{\mathrm{i}}$, for $\mathrm{i} \in \Lambda$, contradiction.

Theorem 2.7: $A \subseteq X$ is open iff $A$ is neighborhood of each $x \in A$.

Proof: Let $A \in \tau, x \in A$. Since we can write $x \in A \subseteq A, A$ is neighborhood of the point $\mathrm{x}$.

Conversely, if $\mathrm{A}$ is neighborhood of each $\mathrm{x} \in \mathrm{A}$, then there exist $\mathrm{U}_{\mathrm{x}} \in \tau$ such that $\mathrm{x} \in \mathrm{U}_{\mathrm{x}} \subseteq \mathrm{A}$. Hence, we have

$\mathrm{A}=\mathrm{U}_{\mathrm{x} \in \mathrm{A}}\{\mathrm{x}\} \subseteq \mathrm{U}_{\mathrm{x} \in \mathrm{A}} \mathrm{U}_{\mathrm{x}} \subseteq \bigcup_{\mathrm{x} \in \mathrm{A}} \mathrm{A}=\mathrm{A}$.

Since the set $A$ is union of the open sets $U_{x}, A$ is open set.

Theorem 2.8: For $x \in X_{\lambda} \subseteq X$ and $x \in A \subseteq X, A$ is neighborhood of $x$ in $X$ such that $A \neq X_{\lambda}$ iff $A \cap X_{\lambda}$ is neighborhood of $x$ in $X_{\lambda}$.

Proof: Let $A$ be a neighborhood of $x$ in $X$. So there is $U \in \tau$ such that $x \in U \subseteq A$. So that $A \cap X_{\lambda}$ is neighborhood of $x$ in $X_{\lambda}$ because of $x \in U \cap X_{\lambda} \subseteq A \cap X_{\lambda}$ and $U \cap X_{\lambda} \in \tau_{\lambda}$.

If $A \cap X_{\lambda}$ is neighborhood of $x$ in $X_{\lambda}$, there is $U \in \tau_{\lambda}$ such that $x \in U \subseteq A \cap X_{\lambda} . U \in \tau$, because of $\tau_{\lambda} \subseteq \tau$. Also we know that $U \subseteq A \cap X_{\lambda} \subseteq A$ and so $A$ is neighborhood of $x$ in $X$.

Theorem 2.9: Let $A \subseteq X$. For $\bar{A}$ is closure of $A, \bar{A}=$ $\bigcup_{\lambda \in \Lambda} \overline{A \cap X_{\lambda}}$.

Proof: Let $x$ be a element of a set $\bar{A}$. Then $x \in K$ such that $K^{c} \in \tau$, for each $K \supseteq A$. Hence $x \in K \cap X_{\lambda}$ such that $\left(K \cap X_{\lambda}\right)^{c} \in \tau_{\lambda}$, for at least one $\lambda \in \Lambda$. So $x \in$ $\mathrm{U}_{\lambda \in \Lambda} \overline{A \cap X_{\lambda}}$.

Let $x$ be a element of a set $\bigcup_{\lambda \in \Lambda} \overline{A \cap X_{\lambda}}$. Then $x \in \overline{A \cap X_{\lambda}}$, for at least one $\lambda \in \Lambda$. So $x \in \bar{A}$ because of $A \cap X_{\lambda}$.

Theorem 2.10: Let $A \subseteq X$. For $A^{\circ}$ is interior of $A$, $A^{\circ}=\bigcup_{\lambda \in \Lambda}\left(A \cap X_{\lambda}\right)^{\circ}$.

Proof: Let $x$ be a element of a set $A^{\circ}$. Then $x \in G$ such that $G \in \tau$, for at least one $G \subseteq A$. Therefore $x \in G \cap X_{\lambda}$, for at least one $\lambda \in \Lambda$ and $G \subseteq A$. So $x \in G \cap X_{\lambda} \subseteq A \cap X_{\lambda}$ such that $x \in G \cap X_{\lambda}$. Thus $x \in \mathrm{U}_{\lambda \in \Lambda}\left(A \cap X_{\lambda}\right)^{\circ}$ and so $A^{\circ} \subseteq \mathrm{U}_{\lambda \in \Lambda}\left(A \cap X_{\lambda}\right)^{\circ}$.

Let $x$ be a element of a set $\bigcup_{\lambda \in \Lambda}\left(A \cap X_{\lambda}\right)^{\circ}$. Then $x \in$ $\left(A \cap X_{\lambda}\right)^{\circ}$, for at least one $\lambda \in \Lambda$. Thus $x \in A^{\circ}$ because of $A \cap X_{\lambda} \subseteq A$. So $\bigcup_{\lambda \in \Lambda}\left(A \cap X_{\lambda}\right)^{\circ} \subseteq A^{\circ}$.

Theorem 2.11: The family $\mathcal{B}=\left\{T \subseteq X: \exists \lambda \in \Lambda, T \in \tau_{\lambda}\right\}$ is the base of $(X, \tau)$ topological space.

Proof: i) We know that for every $\lambda \in \Lambda, X_{\lambda} \in \tau_{\lambda}$ and $\bigcup_{\lambda \in \Lambda} X_{\lambda}=X$

ii) Let $B_{i}, B_{j} \in \mathcal{B}$. So for at least one $i, j \in \Lambda, B_{i} \in \tau_{i}, B_{j} \in$ $\tau_{j}$. For $i \neq j$, the condition is obvious because of $B_{i} \cap$ $B_{j}=\varnothing$. Let $i=j$ and $B_{i} \cap B_{j} \neq \varnothing$. We know that if $B_{i}, B_{j} \in \tau_{i}, \quad B_{i} \cap B_{j} \in \tau_{i}$. So for $B_{i j}=B_{i} \cap B_{j}$, and $x \in B_{i} \cap B_{j}$, there exist $B_{i j} \in \mathcal{B}$ such that $x \in B_{i j} \subseteq B_{i} \cap$ $B_{j}$.

Theorem 2.12: Let the family $\mathcal{B}_{\lambda}$ be a base of $X_{\lambda}$ topological space for $\lambda \in \Lambda$. In this case, $\mathfrak{B}=$ $\left\{T: \exists \lambda \in \Lambda, T \in \mathcal{B}_{\lambda}\right\}$ is the base of $(X, \tau)$ topological space.

Proof: i) Since $\mathcal{B}_{\lambda}$ is the base of $X_{\lambda}$ topological space, it is obvious that $\mathrm{U}_{T \in \mathcal{B}_{\lambda}} T=X$.

ii) For every $\lambda \in \Lambda$, it is obvious that each $\mathcal{B}_{\lambda}$ is discrete. For $i, j \in \Lambda$, let $T_{i} \in \mathcal{B}_{i}, T_{j} \in \mathcal{B}_{j}$ and for $i=j, T_{i} \cap T_{j} \neq \emptyset$.
As $\mathcal{B}_{i}$ is a base of $X_{i}$, for every $x \in T_{i} \cap T_{j}$, there exist $T_{i j} \in \mathcal{B}_{i j}$ such that $x \in T_{i j} \subseteq T_{i} \cap T_{j}$.

Theorem 2.13: The convergent sequence of $X_{\lambda}$ converges in $X$ also.

Proof: Let $\left(x_{n}\right)$ be such a sequence of $X_{\lambda}$ that converges the point $b \in X_{\lambda}$ in $X_{\lambda}$. ( $\left.x_{\mathrm{n}}\right)$ also is a sequence of $X$ because of $X_{\lambda} \subseteq X$. From the definition of convergence we have;

For $\forall T^{\lambda} \in \tau_{\lambda}\left(b \in T^{\lambda}\right), \exists n_{0}^{\lambda} \in \mathbb{N}: n \geq n_{0}^{\lambda} \Rightarrow x_{n} \in T^{\lambda}$.

On the other hand, for $\forall T \in \tau(b \in T)$ we know that $T=T^{\lambda}$ or $T \supseteq T^{\lambda}$. So for $n_{0}=n_{0}^{\lambda}$, if $n \geq n_{0}, x_{n} \in T$. Then $\left(x_{n}\right)$ converges in $X$.

We note that the sequence of $X$ need not be converging in $X_{\lambda}$.

Let us define the set $Z_{G}=\left\{n \in \mathbb{N}: x_{n} \notin G\right\}$ for $\left(x_{n}\right)$ is a sequence of $X$ and $G \in \tau$. Now, we will talk about a different approach for convergence with maks $Z_{G}$. Also, we will take $\operatorname{maks}_{G}=1$ while $Z_{G}=\varnothing$.

Theorem 2.14: Let $\left(x_{n}\right)$ be a sequence of $X$. $\left(x_{n}\right)$ converges the point $b \in X$ iff there exist a $\operatorname{maks} Z_{G}$ for each $G \in \tau(b \in G)$.

Proof: Let $N_{k}=\operatorname{maks}\left\{n_{G}: n_{G}=\operatorname{maks}_{G}, G \in \tau, b \in G\right\}$. Then $\left(x_{n}\right)$ converges the point $b \in X$ In the meanings given in the definition 2.1. So $\left(x_{n}\right)$ converges the point $b \in X$ in the meanings given in the Theorem 2.14 also.

Theorem 2.15: Let $\left(x_{n}\right)$ be a sequence of $X, \lambda, \mu \in \Lambda$, $b \in X_{\lambda}$ and $b^{\prime} \in X_{\mu}$. If $b$ and $b^{\prime}$ is limit point for $\left(x_{n}\right)$, $\lambda=\mu$.

Proof: On the contrary, let $\lambda \neq \mu$. Then $X_{\lambda} \cap X_{\mu}=\emptyset$. Since $\left(x_{n}\right)$ converges the point $b \in X_{\lambda}$, for $n \geq n_{0}$, there exist $n_{0} \in \mathbb{N}$ such that $x_{n} \in X_{\lambda}$. On the other hand, since $\left(x_{n}\right)$ converges the point $b^{\prime} \in X_{\mu}$, for $n \geq m_{0}$, there exist $m_{0} \in \mathbb{N}$ such that $x_{n} \in X_{\mu}$. Let $p_{0}=\operatorname{maks}\left\{n_{0}, m_{0}\right\}$. For $n \geq p_{0}, x_{n} \in X_{\lambda} \cap X_{\mu}$, contradiction.

\section{REFERENCES}

[1] S. Lipschutz,. Schaum's Outline of Theory and Problems of General Topology, McGraw-Hill Book Company, United States of America 1965.

[2] S. Willard, General Topology. Addison-Wesley Publ. Co., Reading, MA 1968.

[3] A. Sutherland Wilson, Introduction to Metric \& Topological Spaces, Oxford University Press 2009. 